\title{
Learning from failure
}

\author{
Elizabeth Loder head of research
}

The BMJ

This week The BMJ is filled with tales of failure, but many would say this is something to celebrate. Failure, and figuring out how to learn from it, is an important part of success, or so the thinking goes. What can be learnt from the failure of high dose folic acid to prevent pre-eclampsia in women at high risk? Results of the FACT trial (doi:10.1136/bmj.k3478) showed no difference in the risk of pre-eclampsia between women who received daily supplements of $4 \mathrm{mg}$ of folic acid and those who received placebo. Our editorialists (doi:10.1136/bmj.k3536) suggest that these negative results teach us that "the concept of a one size fits all prophylactic against pre-eclampsia is unlikely." They call for better phenotyping of the disorder and implementation of proven treatments. These include aspirin prophylaxis, magnesium sulfate, treatment of hypertension, and prompt delivery.

Failure is the explicit focus of another research paper this week. Ben Goldacre and colleagues report (doi:10.1136/bmj.k3218) that half of clinical trials in the European Union Clinical Trials Register failed to report results within 12 months of completion, as required by EU rules. Trials with non-commercial sponsors such as universities or charities were far less likely to report results than trials sponsored by drug or device companies. The online EU Trials Tracker (EU.trialstracker.net) gives detailed information on individual trials and is updated monthly.
This work complements another Goldacre initiative. The "Unreported clinical trial of the week" series was launched in March this year and can be found at BMJ Opinion (http://bit.ly/ 2Qj1THs). Each week this series features an important unreported trial drawn from the US clinicaltrials.gov register. The goal is to shed light on the persistent problem of unreported trials, with the hope of improving reporting rates. What is the lesson here? Negative publicity seems to work: results of some of the highlighted studies have been posted.

Tired of failure? Then you'll enjoy John Middleton's editorial about the successes of the 170 year old Public Health Act for England and Wales (doi:10.1136/bmj.k3653). The list includes slum clearance, better nutrition, improvements in tobacco control, and immunisations. All have contributed to substantial increases in life expectancy.

These are things to be proud of, but the prospect of failure still looms. Middleton reminds us that "human health is improving, but inequalities in and between countries are getting wider and require urgent attention." He draws our attention to the paradox of improvements in human health that have come at the expense of planetary health. Climate change, dwindling numbers of pollinators, shrinking forests, and melting ice caps are all "observable aspects of overproduction and consumption by humans." Time is running out to learn from these failures and to act. 